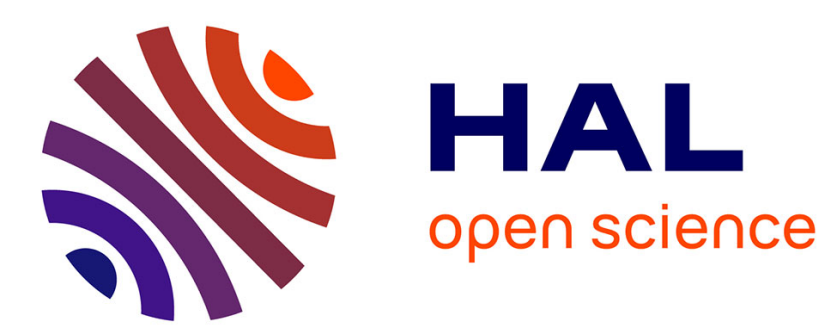

\title{
Lettres d'Émile Benveniste à Claude Lévi-Strauss (1948-1967)
}

John E Joseph, Chloé Laplantine, Georges-Jean Pinault

\section{To cite this version:}

John E Joseph, Chloé Laplantine, Georges-Jean Pinault. Lettres d'Émile Benveniste à Claude LéviStrauss (1948-1967). Histoire Epistémologie Langage, 2020, 42 (1), pp.155-181. 10.1051/hel/2020012 . hal-02952009

\section{HAL Id: hal-02952009 https://hal.science/hal-02952009}

Submitted on 29 Sep 2020

HAL is a multi-disciplinary open access archive for the deposit and dissemination of scientific research documents, whether they are published or not. The documents may come from teaching and research institutions in France or abroad, or from public or private research centers.
L'archive ouverte pluridisciplinaire HAL, est destinée au dépôt et à la diffusion de documents scientifiques de niveau recherche, publiés ou non, émanant des établissements d'enseignement et de recherche français ou étrangers, des laboratoires publics ou privés. 


\title{
LeTtRes D’ÉMILE BENVENISTE A ClaUde LÉVI-STRAuSs (1948-1967)
}

\author{
John E. Joseph ${ }^{1}$, Chloé Laplantine ${ }^{2}$, et Georges-Jean Pinault ${ }^{3}$ \\ ${ }^{1}$ The University of Edinburgh, School of Philosophy, Psychology \& Language Sciences, Edinburgh, UK \\ ${ }^{2}$ CNRS, Histoire des théories linguistiques, Paris, France. \\ ${ }^{3}$ École Pratique des Hautes Études, Paris, France.
}

\section{Résumé :}

Ce travail présente et édite 20 lettres d'Émile Benveniste adressées à Claude Lévi-Strauss entre 1948 et 1967. Il permet de documenter l'histoire du structuralisme, du dialogue et du rapprochement de la linguistique et de l'ethnologie, par exemple dans les discussions concernant la parenté ou l'organisation sociale, ou celles accompagnant la création de la revue L'Homme.

Mots-clés :

Benveniste, Lévi-Strauss, ethnologie, histoire du structuralisme

\section{Abstract:}

We here provide a presentation and edition of 20 letters sent by Émile Benveniste to Claude Lévi-Strauss between 1948 and 1967. They shed new light on the history of structuralism, the dialogue between linguistics and ethnology, for example in the discussions concerning kinship or social organization, or surrounding the creation of the journal L'Homme.

Keywords:

Benveniste, Lévi-Strauss, ethnology, history of the structuralism

Nous publions ici les lettres d'Émile Benveniste à Claude Lévi-Strauss ${ }^{1}$, conservées par ce dernier et qui sont consultables (sous réserve d'autorisation) à la Bibliothèque nationale de France dans le Fonds Claude Lévi-Strauss sous la cote NAF $28150(183)^{2}$. Malheureusement il n'a pas été possible de publier les lettres de Lévi-Strauss à Benveniste, toute la correspondance reçue par Benveniste ayant disparu ${ }^{3}$. La correspondance publiée ici (et qui est possiblement incomplète) débute en 1948, aux lendemains de la soutenance de la thèse de doctorat d'État de Lévi-Strauss ${ }^{4}$, par une discussion concernant l'organisation de la parenté indo-européenne, et se poursuit jusqu'en 1967. D'après la correspondance Jakobson / LéviStrauss publiée par Emmanuelle Loyer et Patrice Maniglier et complétée par Pierre-Yves Testenoire ${ }^{5}$, où il est souvent question de Benveniste, les deux hommes se rencontrent en 1946, et commencent à échanger en $1947^{6}$ dans le contexte de l'écriture de la thèse sur la parenté,

\footnotetext{
${ }^{1}$ Françoise Bader (2012) dans un article portant également sur cette archive, publiait quatre des vingt lettres de Benveniste et en débutait l'analyse du point de vue de l'histoire intellectuelle. Le présent travail édite l'intégralité des lettres et apporte un appareil de références plus complet.

${ }^{2}$ Nous remercions ici Monique Lévi-Strauss de nous avoir autorisés à consulter et à publier cette archive, ainsi que Michel Zink, Secrétaire perpétuel de l'AIBL, de nous avoir autorisés à publier les lettres d'Émile Benveniste.

${ }^{3}$ Pour être précis, il n'est pas exclu qu'une partie de cette correspondance soit actuellement entre des mains privées, mais nous n'avons pas identifié les détenteurs de ces précieux documents.

${ }^{4}$ Lévi-Strauss soutient son doctorat d'État le 5 juin 1948 (thèse principale : Les Structures élémentaires de la parenté ; thèse complémentaire : "La vie familiale et sociale des Nambikwara 》. Le directeur de thèse est un sociologue durkheimien, Georges Davy, et le jury est composé d'Albert Bayet (sociologue), Émile Benveniste, Jean Escarra (juriste sinologue), et Marcel Griaule (ethnologue africaniste).

${ }_{6}^{5}$ Jakobson et Lévi-Strauss (2018), et Testenoire (2019).

${ }^{6}$ Lettre du 29 décembre 1947 de Lévi-Strauss à Jakobson : « nous avons passé deux heures à une discussion si animée du système de parenté indo-européen que ni [Benveniste] ni moi n’avons plus pensé à la question [de la
} 
Benveniste apportant à Lévi-Strauss sa connaissance du domaine indo-européen ${ }^{7}$. En effet, de 1945 à 1952 Benveniste poursuit au Collège de France ${ }^{8}$ un enseignement sur le « vocabulaire des institutions indo-européennes », développant chaque année une étude particulière de tel ou tel domaine institutionnel. Ces études seront publiées en 1969 dans l'ouvrage du même nom ${ }^{9}$ et la question de la parenté indo-européenne y occupe un long chapitre ${ }^{10}$; elle fait également l'objet d'un article spécifique publié en 1965 dans L'Homme ${ }^{11}$.

Au dire de Lévi-Strauss (se confiant à Jakobson), Benveniste était le seul membre de son jury de thèse à même de suivre la démarche d'analyse développée dans les Structures élémentaires : « Je ne vous parle pas de la soutenance : ce fut une longue corvée, et Benveniste a été le seul membre du jury à comprendre ce que j'ai voulu faire ${ }^{12} »$. Cette compréhension mutuelle ne se limite pas à la thématique de la parenté dont ils traitent tous les deux, mais elle tient à leur manière de l'aborder. En effet, à plusieurs reprises Benveniste confie à Lévi-Strauss qu'il entrevoit dans l'approche structurale qui guide ses recherches un avenir ouvert pour les sciences humaines : "Je ne sais quel accueil y feront les ethnologues qualifiés, mais je crois que tôt ou tard la méthode d'analyse structurale s'imposera ici comme ailleurs et que vous aurez le mérite d'avoir ouvert une voie neuve. Votre ouvrage alimentera les discussions les plus fécondes, d'où non seulement ce problème, mais quantité d'autres sortiront transformés ». Ailleurs, Benveniste conçoit la « structuralisation » comme une « des qualités que l'ethnologie est en train d'acquérir ${ }^{13} »$.

Le structuralisme n'a jamais eu une définition unanimement acceptée. Celui de LéviStrauss, relativement constant, ne coïncide pas avec celui de Benveniste, toujours en évolution depuis les années 1930 jusqu'à la fin des années 1960, quand il n'est plus évident qu'il reste structuraliste du tout ${ }^{14}$. Son enthousiasme pour la démarche de Lévi-Strauss est donc assez surprenant, mais témoigne de sa capacité à apprécier un type d'analyse qui n'est pas le sien mais qui rend possible une percée scientifique dans un champ où il a un vif intérêt - l'analyse culturelle - et ne réclame pas une unique autorité. Comme Benveniste, Lévi-Strauss rapprochait ethnologie et linguistique, contre la pratique traditionnelle qui reléguait la linguistique aux marges. Dans le cas de Benveniste, on doit identifier sa fidélité à un héritage intellectuel au moins double, d'une part l'enseignement de Meillet, relatif à la dimension sociologique de la linguistique, et d'autre part la lecture de Marcel Mauss, qui orientait davantage vers l'anthropologie.

participation du Cercle linguistique de New York à un Congrès de linguistique] ». Jakobson et LéviStrauss (2018: 75).

${ }^{7}$ Lévi-Strauss à Jakobson le 4 juillet 1948 : « Benveniste et moi échangeons une longue correspondance sur des problèmes de parenté hindous, iraniens et grecs ; nous nous entendrions très bien s'il n'était si défiant et cérémonieux. C'est au compte-gouttes, et avec mille précautions, qu'il se décide, peu à peu, à me communiquer un renseignement ou à me consulter sur un problème. Enfin, ces relations épistolaires contribuent à la préparation de la suite de mon travail ». Jakobson et Lévi-Strauss (2018: 93). Dans la section des Structures élémentaires (Lévi-Strauss [1949a] 1967 [2 édition] : 454-547) consacrée au système de la parenté en Inde et dans le monde indo-européen, Lévi-Strauss fait référence (459 n. 17, 545 n. 33) à deux articles de Benveniste (Benveniste 1932 et 1938).

${ }^{8}$ Benveniste occupe la chaire de « Grammaire comparée » depuis 1937.

${ }^{9}$ Ce qui aboutira aux deux volumes publiés en 1969 : Benveniste (1969a) et (1969b).

${ }^{10}$ Benveniste (1969a : 203-276).

11 Benveniste ([1965] 2015 : [5-16] 291-303).

${ }^{12}$ Lettre du 4 juillet 1948 : Jakobson et Lévi-Strauss (2018 : 93). Même propos avant même la soutenance dans sa lettre du 6 mars 1948 : "j'ai intrigué pour que Benveniste fasse partie du jury, et j'attache du prix à sa présence, car il sera sans doute le seul à comprendre ce que j’ai voulu faire ». Jakobson et Lévi-Strauss (2018 : 86).

${ }^{13}$ Lettre 6, du 22 Janvier 50.

${ }^{14}$ La question du « structuralisme » dans les sciences humaines dépasse le cadre du présent article, et n'est donc pas traitée ici de manière détaillée. 
Un mois après sa soutenance de thèse, Lévi-Strauss rapporte à Jakobson une discussion à propos de la comparaison des structures linguistiques (sud-asiatiques, indo-européennes, américaines) et des structures de la parenté : Benveniste «considère le parallélisme sans signification parce que, dit-il, la structuration n'existe dans la langue qu'au niveau des éléments différentiels et qu'on ne peut la retrouver au niveau de la grammaire ou du vocabulaire. Il ne pense donc pas qu'il y ait des structures formelles coextensives au champ entier de la pensée inconsciente ${ }^{15}$ ». Dans son texte « L'analyse structurale en linguistique et en anthropologie ${ }^{16}$ » publié en 1945 dans le premier numéro de la revue Word et qui fonde l'approche structurale en ethnologie, ou dans "Histoire et ethnologie ${ }^{17}$ », Lévi-Strauss fait de la reconnaissance du caractère inconscient des faits linguistiques et sociaux une base essentielle de l'analyse structurale $^{18}$, et c'est dans la phonologie de Troubetzkoy et surtout dans les propos sur l'inconscient linguistique formulés par Boas dans 1'Introduction au Handbook of American Indian Languages ${ }^{19}$ qu'il voit l'émergence de ce principe d'analyse. Benveniste se montre enthousiaste concernant les réflexions de Lévi-Strauss - «Est-il besoin de vous dire que j'adhère à ce que vous dîtes du caractère inconscient des faits linguistiques et sociaux. Vous devriez une fois traiter en détail le problème du symbolisme dans les faits sociaux ${ }^{20} \gg-$, mais lorsqu'il parle de l'inconscient dans un article qu'il a écrit pour une nouvelle revue lancée par Jacques Lacan (La Psychanalyse), ce sera dans un cadre freudien; et quant au social, Benveniste insistera dans ses derniers écrits et ses dernières conférences sur le fait que «la langue contient la société », comme il le démontre à chaque page du Vocabulaire des institutions indo-européennes, et sur le fait que les autres systèmes sémiologiques ont besoin de la langue comme interprétant. Ce ne sont pas les préoccupations de Lévi-Strauss ; on ne peut parler ni d'une dispute, ni même d'un dialogue. L'analogie la plus apte est peut-être avec les « monologues collectifs » de Piaget.

Lévi-Strauss joue un rôle dans la mise en œuvre de certains projets de Benveniste en Amérique du Nord : il le met en relation en février 1950 avec Edward D'Arms de la Fondation Rockefeller $^{21}$; cette institution soutiendra son invitation à enseigner au Summer Institute de la Linguistic Society of America à Ann Arbor de juin à août 1950, un projet de conférence américano-européenne sur la sémantique (1950-1951), et deux séjours de plusieurs mois sur la côte Nord-Ouest où Benveniste enquêtera sur différentes langues : Haida, Tlingit, Gwich'in, Inupiaq (1952 et 1953) ${ }^{22}$.

Les lettres publiées ici font aussi référence à la collaboration des deux hommes autour de la revue L'Homme. Revue française d'anthropologie (fondée par Benveniste, Pierre

\footnotetext{
${ }^{15}$ Lettre du 4 juillet 1948. Jakobson et Lévi-Strauss (2018 : 97). Voir Lévi-Strauss (1951) pour la publication de cette hypothèse de comparaison entre structures de parenté et structures linguistiques, et ici la lettre 9 , et la note 57.

${ }^{16}$ Lévi-Strauss ([1945] 1958 et $1974:$ 43-69).

17 Qui devient plus tard le chapitre d'introduction d'Anthropologie structurale. Lévi-Strauss ([1945] 1958 et 1974 : 9-39).

${ }^{18}$ Voir aussi l'introduction au livre de Mauss Sociologie et anthropologie, Lévi-Strauss 1950b : IX-LII. Voir aussi Laplantine (2018 : 25-26, et 2011 : 93-97).

${ }^{19}$ Boas (1911 : 67-73). Lévi-Strauss attribue à Boas « le mérite d'avoir, avec une admirable lucidité, défini la nature inconsciente des phénomènes culturels, dans des pages où, les assimilant de ce point de vue au langage, il anticipait sur le développement ultérieur de la pensée linguistique, et sur un avenir ethnologique dont nous commençons à peine à entrevoir les promesses » Lévi-Strauss ([1945] 1958 et 1974 : 32). Notons que LéviStrauss participe grandement à faire connaître Boas en France, en traduisant notamment de longs passages de cette « Introduction ».

${ }^{20}$ Lettre 6, du 22 Janvier 50.

${ }^{21}$ Archives de la Rockefeller Foundation : RF, RG 1.2, Series 500R, Box 12, f. 112.

${ }^{22}$ Voir la lettre 10, du 14 novembre 1953.
} 
Gourou $^{23}$ et Lévi-Strauss) et du long processus de sa mise en route. Il en est question dès $1949^{24}$; le premier numéro de la revue paraîtra en 1961. C'est une revue d'anthropologie originale en ce qu'elle donne une place importante aux approches linguistiques de la culture. C'est dans cette revue que paraîtra en 1962 l'article de Jakobson et Lévi-Strauss sur Les Chats de Baudelaire, que Benveniste a relu en donnant à Lévi-Strauss une liste de commentaires qui seront publiés partiellement en note de l'article, et qu'on reproduit ici intégralement (21). Quelques années plus tard, en 1967, Benveniste entreprendra sa recherche sur « la langue de Baudelaire " en distinguant sa démarche, la recherche d'une structure profonde, de celle de Lévi-Strauss et Jakobson ${ }^{25}$ qui abordent le poème comme un objet et le démontent en révélant ses structures formelles. Benveniste s'intéresse à la manière dont est fait le poème, mais en même temps cette recherche n'est pas dissociée de la question de ce que fait le poème, mettant le sujet au cœur de l'analyse ${ }^{26}$.

Dans l'hommage qu'il rend à Benveniste en 1976 dans L'Homme - et cet hommage prend tout son sens en ce qu'il est publié dans ce journal et en rappelle le projet initial -, LéviStrauss n'est pas loin de dire que le Vocabulaire des institutions indo-européennes est un livre d'ethnologie et que Benveniste est un ethnologue des langues et du langage : "Cet illustre spécialiste des langues indo-européennes portait un vif intérêt à l'ethnologie, comme en témoignent la place non négligeable qu'occupent dans ses travaux des langues africaines et amérindiennes - ces dernières, étudiées par lui sur le terrain en Colombie britannique et en Alaska - et les enseignements ethnologiques que, tout au long de sa vie, il ne cessa de demander à la philologie comparée. A cet égard, il n'est pas excessif de dire que son ouvrage en deux volumes sur le Vocabulaire des institutions indo-européennes apporte à l'anthropologie sociale une contribution d'importance majeure. Cette orientation constante explique qu'en 1948, il ait bien voulu accepter de siéger au jury de soutenance de ma thèse sur les structures élémentaires de la parenté27 $»$.

(1)

1 rue Monticelli (14e)

Paris le 5 Juin 48

Cher Monsieur,

Que de ma courte et bien inutile intervention à votre soutenance il reste au moins deux ou trois remarques sur des points de détail. Vous trouverez sur la feuille jointe ${ }^{28}$ le texte dont je me suis servi et l'indication de la source. En outre, il y aurait lieu de rectifier en partie ce que vous dites des sapinda (p. 684 sq.). Le mot pinda ne signifie pas "corps" ou il n'a cette acception que tardivement et par transposition métaphorique du seul et vrai sens qui est

\footnotetext{
${ }^{23}$ Pierre Gourou (1900-1999) est un géographe spécialiste de l'Indochine et de la géographie tropicale. Sur Pierre Gourou, on peut se référer à l'article de Michel Bruneau (2000).

${ }^{24}$ Lettre 4, du 21 avril 49.

25 « Différences d'approche / Une approche consiste à partir de lacompesition poéti < de la pièce de > vers comme d'une donnée, de la décrire, de la démonter comme un objet. C'est l'analyse telle qu'on la trouve appliquée aux Chats dans le bel article de Lévi-Strauss et Jakobson. / Une autre approche eonsistera en une sera d'un type tout autre. On s'efforcera d'atteindre la structure profonde de son univers poétique dans le choix révélateur des images et dans leur articulation ». Benveniste (2011: 186).

${ }^{26}$ En effet, Benveniste insiste sur les « émotions », comme base des images poétiques, autrement dit sur le corps du poète, voir Benveniste $(2011: 26,28,192)$, et en particulier : « Le discours poétique est un discours d'émotion » (Benveniste $2011: 246)$.

${ }^{27}$ Lévi-Strauss (1976:5).

${ }^{28}$ Cette feuille n'a pas été retrouvée dans le fonds de la BnF.
} 
"boulette d'offrande 29 ". Les sapinda sont ceux qui sont unis par l'obligation de l'offrande cultuelle du pinda. Vous pourriez donc, si vous êtes encore maître de votre manuscrit, faire l'économie <au bénéfice de votre démonstration> de toute la page où vous essayez d'interpréter sapinda en fonction du sens fictif de "corps".

J'aurais aussi quelques réserves à faire sur l'assimilation $<(\mathrm{p} .675 \mathrm{sq})>$ des "couleurs" des castes ou classes aryennes avec les (Os) blancs et noirs ${ }^{30}$. J'ai l'impression qu'il s'agit de choses différentes. Les "couleurs" aryennes sont emblématiques de classes ou d'états, non de fractions tribales ou de "moitiés". Il ne faudrait pas non plus tirer du texte indien que vous citez (je n'ai malheureusement plus votre ms.) une équivalence trop précise entre l'opposition ārya / dāsa et celle blanc / noir. La couleur qui est ici celle du visage n'est qu'indirectement impliquée dans une opposition qui n'est, littéralement prise, qu'ethnique.

Enfin sur les transcriptions de mots sanskrits : écrire gotra (sans signe de longue) et svayamvara (sans signe de longue non plus).

Un mot encore. La position que vous prenez vous donne incontestablement le droit de juger vos devanciers. La justice coïncide-t-elle avec le jugement qui énonce «Malinowski, avec la légèreté qui le caractérise ...»? L'œuvre de Malinowski n'est-elle vraiment $<,>$ caractérisée $<$ "> que par la légèreté ?

Mes félicitations encore pour votre brillante soutenance et mes remercie-/ments pour les indications relatives au Handbook de Boas. ${ }^{31}$

Croyez-moi, je vous prie, votre cordialement dévoué,

EBenveniste

1 rue Monticelli $\left(14^{\mathrm{e}}\right)$

Paris le 16 juin 1948

Cher Monsieur,

Pour répondre à votre question, il m'a fallu me reporter à Herzfeld ${ }^{32}$ et à divers ouvrages que je n'avais pas sous la main. La liste de Videvdad (ou Vendidad) XII pose au moins en effet une question qui demandait examen et quand je vous ai donné la traduction généralement admise des termes de parenté (d'après Bartholomae ${ }^{33}$ ), je n'ai pas tenu compte d'une discordance qui se manifeste entre le sanskrit et l'avestique dans l'acception d'un terme.

Voici donc les termes dans leur séquence exacte et dans leur forme :

\begin{tabular}{|c|c|}
\hline Vd. XII, I & « père ; mère » \\
\hline $3-4$ & $p u \theta r \bar{o} \ldots d u \gamma \delta a$ \\
\hline $5-6$ & brāta ... x $x^{v} a \eta h a$ \\
\hline $7-8$ & 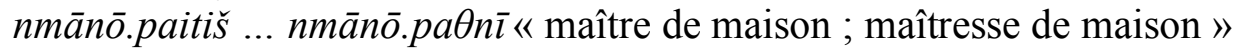 \\
\hline $9-10$ & nyāka ... nyāke 《 grand-père ; grand-mère » \\
\hline $11-12$ & «petit-fils ; petite-fille » \\
\hline $13-14$ & brātruyō ... brātruye « fils du frère ; fille du frère » \\
\hline $15-16$ & tüiryo ... tüirya $\quad$ "frère du père ; sœur du père » \\
\hline
\end{tabular}

\footnotetext{
${ }^{29}$ Comparer désormais Lévi-Strauss (1967 : 462-464 et 481-482, spécialement 463).

${ }^{30}$ Voir désormais Lévi-Strauss (1967: 458).

${ }^{31}$ Boas (1911).

${ }^{32}$ Herzfeld 1947. Ernst Herzfeld (1879-1948), archéologue, philologue et historien, fut une figure importante des études du Proche-Orient et de l'Iran ancien ; professeur à Berlin à partir de 1920, contraint de s'exiler en 1933 du fait de son ascendance juive, puis professeur à Princeton (Institute for Advanced Studies) à partir de 1936. Voir Hauser (2003 [2012]).

${ }^{33}$ Bartholomae (1904).
} 


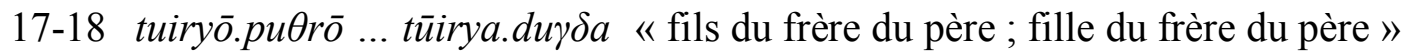

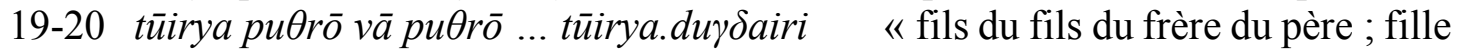
$\mathrm{du}$ fils du frère du père »

Voilà la liste des nabānazdišta (les plus proches du nombril ou par le n. ${ }^{34}$ )

1) Il faut observer que en skr. bhrātrvya signifie certainement « fils du frère du père » (cousin parallèle), mais ce sens ne peut être admis en iranien bien que la forme soit identique ${ }^{35}$. Vous observerez que s'il fallait calquer le sens de av. brātruya sur skr. bhrātrvyya, on ne distinguerait plus brātruyō du terme tüirya puӨra qui désigne sûrement le « fils du frère du père ». Il faut donc admettre que brātuirya a été ramené à "fils du frère » et c'est ce que confirment les formes / modernes comme pašto wrōra « fils du frère ${ }^{36}$ ». Herzfeld a donc raison de rendre brätuirya par «brother's son ». Mais attention : il cite (p. 112 en haut) comme preuves skr. bhrātrvya et lat. fratruus. Or skr. bhrätrvya a un tout autre sens et lat. «fratruus » n'existe pas. C'est malheureusement souvent ainsi chez Herzfeld.

2) L'expression complexe de 19-20 doit avoir très vraisemblablement le sens que je lui donne pour le premier terme (masculin) tüirya puӨrō vā puӨrō bien que la langue soit bizarre ;

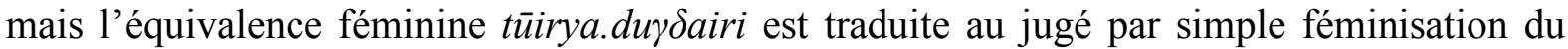
terme précédent. Il n'y a pas d'autre exemple du mot $d u \gamma \delta a i r i{ }^{37}$. Il faut donc décider entre « fille du fils du frère du père » et « fille de la fille du frère du père », ce qui est différent. Votre expérience de ces problèmes vous inspirera sans doute un avis.

Vous avez sans doute raison pour le texte de la Mimāṃsa [sic ${ }^{38}$. Il m'est soudain revenu en mémoire la veille de votre soutenance. Je l'ai copié hâtivement et sans reproduire les signes de longues qui distinguent deux noms propres et le relisant le matin, il m'a semblé poser une question dont je vous ai fait part ${ }^{39}$. Mais Vāsudeva est certainement fils de Vasudeva et le problème est tout autre comme vous le dites.

Il faut donc se résigner, comme je le vois, à ne retenir de Malinowski, que les données d'observation. C'est signe, après tout, que l'ethnologie se rigorise. Votre beau travail en est la preuve.

Croyez-moi, cher Monsieur, votre bien dévoué, EBenveniste

(3)

1 rue Monticelli (14e)

Paris le 24 juin $1948^{40}$

Cher Monsieur,

\footnotetext{
${ }^{34}$ Littéralement, "les plus proches par la parenté" (Bartholomae $1904: 1040$ ) ; le premier terme $n a b \bar{a}^{\circ}$ est apparenté à av. $n \bar{a} f a-, n \bar{a} f a h-$ « parenté, famille », originellement « nombril », cf. véd. nābhi- « nombril, moyeu, centre, origine, parenté »; voir Mayrhofer 1956-1976, II : 135, 153 et 1986-1996, II : 13-14, avec références. ${ }^{35}$ Discussion reprise dans Benveniste (1969a : 259-261 et 264-265), dans le chapitre « Formation et suffixation des termes de parenté ». Scénario de Benveniste contesté par Szemerényi (1977 : 62-63) ; discussion et autres références dans Mayrhofer (1986-1996, II : 281).

${ }^{36}$ La dactylographie est sûre pour wrōro. Il faut cependant lire wrāra, cf. Morgenstierne (1927: 89, n² 277) ( wrāra 'nephew') et Morgenstierne (2003 : 91) (idem, 'nephew, brother's son'. Le nom pašto pour « frère » est wror, hérité de l'étymon iranien, et le terme wrāra est rapproché d'av. brātruya-, comme le reprend Benveniste. ${ }^{37}$ Sur ce terme, qui est dérivé du nom avestique de la fille (durdar-), voir Szemerényi (1977 : 58 n. 222).

${ }^{38}$ Il faut évidemment lire Mīmāṃsā.

${ }^{39}$ Texte du Tantravārttika sur le mariage d'Arjuna et Vāsudeva, traduit par Renou (1961 : 213-214). Voir LéviStrauss (1949 [1967] : 466, n. 54).

${ }^{40}$ Cette lettre comporte des mots grecs écrits en marge au crayon, sans accent ni esprit ( $« \varepsilon \delta v o v / \gamma \alpha \mu \varepsilon v$ / $\eta \gamma \varepsilon 1 \sigma \theta \alpha$ / $\varepsilon \gamma \gamma v \eta \sigma i \varsigma », ~ « \varepsilon \delta v o v »)$, mais ils ne sont pas de l'écriture de Benveniste. Ils sont probablement dus à la personne qui a eu les papiers en main et qui les a triés à un certain moment.
} 
Il me semble que la manière dont vous appréciez les relations des nabānazdišta rend bien compte, à l'intérieur de la structure iranienne, de cette exogamie régulièrement contaminée d'endogamie qui caractérise la société avestique, au moins dans ses représentants nobles $^{41}$. La filiation patrilinéaire est certaine. Le point intéressant est de pouvoir faire ressortir, par l'analyse des relations de parenté, la fréquence des mariages consanguins.

J'ai cherché à préciser le problème des nabānazdišta, mais n'ai pu découvrir malgré mes recherches de texte plus explicite que celui qui vous occupe. Il reste un problème, mais qui ne comporte pas en ce moment, pour la même raison, de solution : celui de la dénomination de nabānazdišta. Celle-ci est purement iranienne : la forme correspondante du skr. n'est attestée que dans un nom propre Nabhānedișthah et n'enseigne rien ${ }^{42}$. On est conduit à se demander si ce n'est pas là la désignation spécifique de la parenté telle qu'elle résultait de ce type de mariage. On ne peut que poser la question.

Un autre point mérite l'attention, surtout à cause de faits connexes qu'il évoque. Nous avons affaire à une parenté qui se manifeste à l'occasion du deuil. Or je suis intrigué depuis un certain temps par un fait singulier de la terminologie grecque, qui est sans parallèle à ma connaissance. En grec, un même terme ${ }^{43}$ désigne la parenté par alliance et le deuil. La notion de rendre les derniers devoirs et celle de s'allier par un mariage ont la même expression. Estce le reflet d'une exogamie stricte, selon laquelle le clan où je prends femme assume l'obligation d'ensevelir mes morts à charge de réciprocité ? Connaît-on d'autres exemples de prestations funéraires aussi rigoureusement liées à l'échange de femmes ?

Il y a quelques jours, avant d'avoir reçu votre lettre, j'ai eu l'occasion de voir M. Dumézil ${ }^{44}$ et d'évoquer avec lui les noms de ceux qui pourraient assumer les enseignements vacants à la $\mathrm{V}^{\mathrm{e}}$ section. Le vôtre a naturellement été prononcé, / et j'ai dit ce que je pensais d'une œuvre que M. Dumézil n'était pas encore à même de connaître, mais sur laquelle son sentiment sera, on peut le présumer, favorable.

Veuillez me croire, cher Monsieur, votre bien dévoué, EBenveniste

(4)

Collège de France

Chaire de Grammaire comparée
Paris, le 21 avril 49

1 rue Monticelli $\left(14^{\mathrm{e}}\right)$

Cher Monsieur,

La question que vous voulez bien me poser, et qui a de suggestifs prolongements, ne laisse pas de m'embarrasser. Vous savez qu'on a beaucoup discuté sur la forme du $\delta \varepsilon \dot{\pi} \alpha \varsigma$ $\dot{\alpha} \mu \varphi \kappa v ́ \pi \varepsilon \lambda \lambda$ ov, et que les <commentateurs> anciens, non plus que les modernes, n'en avaient probablement jamais $\mathrm{vu}^{45}$. Je ne puis que vous indiquer deux précisions de fait.

\footnotetext{
${ }^{41}$ Allusion à cette pratique dans Lévi-Strauss (1967 : 545). Voir désormais Skjaervø (2013).

${ }^{42}$ En fait, forme de ce nom propre en védique est $N a \bar{b} b h a ̄ n e ́ d i s t h a-$ (nom d'un poète), dérivé patronymique, qui signifie donc « descendant d'un proche parent », *nabhā-nediștha-= avestique nabā-nazdista-. Voir Mayrhofer (2003: 50, 2.1.272).

${ }^{43}$ Il s'agit de $\kappa \tilde{\eta} \delta o \varsigma$ (depuis Homère) “soin, souci”, qui s’est spécialisé dans les deux emplois mentionnés par Benveniste : « deuil, honneurs rendus à un mort » (Hom., ion. -att.) et « union, parenté par mariage, par alliance » (Eschyle, Hérodote), sens qui se retrouve dans plusieurs dérivés, voir Chantraine (1968-1980 : 523); Beekes (2010 : 684).

${ }^{44}$ Georges Dumézil (1898-1986) était directeur d'études à la Ve section (Sciences religieuses) de l'École Pratique des Hautes Études. Il occupera la chaire des civilisations indo-européennes au Collège de France de 1949 à 1968, puis, soutenu par Lévi-Strauss, sera élu à l’Académie française en 1978.

${ }^{45}$ On pourrait être tenté de mettre en relation la curiosité de Lévi-Strauss pour ce «vase à double coupe » avec sa fascination répétée pour les choses doubles, jumelles, scindées. Cf. par exemple Lévi-Strauss (1991).
} 
1) L'expression se retrouve chez Homère en bien d'autres endroits que les quatre que

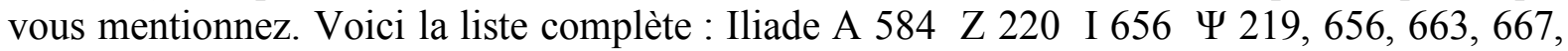
699 - Odyssée $\gamma 63$ o 102, 120 v 57 cf. $\theta 89 \chi 86$ v 183. Il faudrait en outre tenir compte de l'expression, qui a été considérée comme synonyme, pió $\lambda \eta \dot{\alpha} \mu \varphi \dot{i} \theta \varepsilon \tau 0 \varsigma \Psi 270,616$. /

2) $\dot{\alpha} \mu \varphi \iota \kappa v ́ \pi \varepsilon \lambda \lambda$ o signifie littéralement « qui est pourvu, de part et d'autre ( $\dot{\alpha} \mu \varphi \imath)$, d'une coupe ( coupe faite de deux récipients accolés » ? Mettons, si vous voulez, un récipient $<$ à boire $>$ formé de deux coupes accolées ${ }^{47}$. Voilà à peu près ce que le sens littéral permet d'imaginer. Mais comment se représenter matériellement l'objet? L'avis d'un helléniste archéologue (M. Chapouthier par exemple) ${ }^{48}$ serait précieux. Il faudrait connaître l'état actuel d'un débat qui n'a pas dû cesser chez les archéologues et sur lequel je n'ai pas d'information.

Voilà le peu que je me hasarde à vous dire, regrettant de ne pouvoir être plus affirmatif. Mais l'idée d'une relation avec des cultes est ingénieuse et mériterait d'être poursuivie si les données matérielles autorisent une représentation précise. /

Je dois me rendre en Angleterre la première semaine de Mai, mais pour un court séjour $^{49}$. A mon retour, vers le milieu du mois, nous pourrons nous entretenir de l'état de la revue.

Croyez, cher Monsieur, à mes cordiaux sentiments, EBenveniste

(5)

Collège de France

Chaire de grammaire comparée
Paris, le 6 Août 49

1 rue Monticelli $\left(14^{\mathrm{e}}\right)$

Cher Monsieur,

Je veux, d'un mot au moins, vous dire combien j'ai trouvé d'intérêt à relire, cette fois « objectivés » par la forme imprimée, vos deux travaux, la description si suggestive des Nambikwara où une analyse très stricte ne fait pas tort à la sympathie humaine, et surtout le beau livre sur la parenté. Je ne sais quel accueil y feront les ethnologues qualifiés, mais je crois que tôt ou tard la méthode d'analyse structurale s'imposera ici comme ailleurs et que vous aurez le mérite d'avoir ouvert une voie neuve. Votre ouvrage alimentera les discussions les plus fécondes, d'où non seulement ce problème, mais quantité d'autres / sortiront transformés.

Avec tous mes souhaits pour le développement de vos recherches et mes sentiments cordiaux,

\section{EBenveniste}

(6)

Collège de France

Chaire de Grammaire comparée
Paris, le 22 Janvier 50

1 rue Monticelli $14^{\mathrm{e}}$

\footnotetext{
${ }^{46}$ Interprétation littérale, cf. Chantraine (1968-1980 : 600) et (Beekes $\left.2010: 804\right)$, avec références.

${ }^{47}$ Cette interprétation était celle d'Aristote (Histoire des Animaux, 624a), qui considérait le vase en question comme une coupe dont le pied creux forme lui-même une coupe. Il y comparait les cellules des ruches d'abeilles.

${ }^{48}$ Fernand Chapouthier (1899-1953), professeur de langue et littérature grecques en Sorbonne, et directeur adjoint de l'École normale supérieure.

49 Allusion à la mission de Benveniste en mai 1949, pour donner des conférences à l'université de Londres, cf. Annuaire du Collège de France, année 1948-1949, p. 159.
} 


\section{Cher Monsieur,}

J'ai lu avec grand intérêt les deux travaux que vous m'avez aimablement communiqués. Dans le Southwestern J. of Soc. l'article de Capell est riche de faits instructifs que j'aimerais discuter par écrit et en tout cas utiliser ${ }^{50}$. Mais pour l'instant je n'en ai pas le loisir.

Le travail de Garvin sur le Ponape est un bon exemple de monographie strictement descriptive et conduite selon la méthode bloomfieldienne orthodoxe ${ }^{51}$. C'est une étude de bonne qualité, encore qu'elle ne soit pas complète, surtout dans la partie syntaxique. Le problème me paraît surtout pratique et d'ordre financier : une étude longue et d'impression coûteuse ne risque-t-elle pas / d'obérer dangereusement les ressources de L'Homme dès le début ? Et si on la mettait en réserve pour un des exercices à venir, l'auteur accepterait-il de se voir renvoyé à une date imprévisible?

Je me suis demandé si on ne pourrait pas se borner à une partie seulement, en retranchant p. ex. les conversations-phonogrammes, utiles mais vraiment longues et les portions trop techniques. Mais ce serait peu seyant et l'auteur n'accepterait probablement pas.

Vous avez mon avis - ou plutôt mes inquiétudes. J'ai peur que cette dépense, si on l'entreprend, ne paralyse pour longtemps l'effort de l' « Homme».

Je vous dépose les deux travaux à l'Ecole.

Croyez-moi, cher Monsieur, votre bien cordialement dévoué, EBenveniste

Lundi soir. J'ai trouvé au Collège votre article de la Rev. de Métaph.$^{52}$, dont je vous remercie. Vous posez avec / lucidité un problème de grande portée. La critique de Malinowski est pertinente. Mais je ne suis pas certain que vous ne fassiez pas l'histoire plus belle qu'elle n'est, en lui prêtant implicitement quelques unes des qualités que l'ethnologie est en train d'acquérir - la structuralisation et tout ce qui en découle ${ }^{53}-$, de quoi les historiens actuels me paraissent loin d'avoir pris conscience. - Est-il besoin de vous dire que j'adhère à ce que vous dîtes du caractère inconscient des faits linguistiques et sociaux ${ }^{54}$. Vous devriez une fois traiter en détail le problème du symbolisme dans les faits sociaux.

Cher Monsieur,

\footnotetext{
${ }^{50}$ Capell (1949 : 169-189).

${ }^{51}$ Paul L. Garvin a fait circuler ses diverses études sur la langue ponapéenne sous forme de miméographe (par ex. « A Linguistic Study of Ponapean » et «A Definitional Grammar of Ponapean », que Kenneth L. Rehg et Damian G. Sohl déclarent « invaluable » dans la préparation de leur propre Ponapean Reference Grammar, Honolulu : University of Hawaii Press, 1981), mais elles sont restées inédites. Celle à laquelle Benveniste réagit est sans doute « Ponapean, a Micronesian Language », dont un compte rendu cinglant par Aurélien Sauvageot est paru dans le Journal de la Société des Océanistes. Voir Sauvageot (1951 : 315-317).

${ }^{52}$ Lévi-Strauss 1949b (« Histoire et ethnologie »).

${ }^{53}$ Voir par exemple dans cet article : « On dira peut-être que ces malencontreuses incursions dans le domaine de la sociologie comparée sont, après tout, des exceptions dans l'œuvre de Malinowski. Mais l'idée que l'observation empirique d'une société quelconque permet d'atteindre des motivations universelles y apparaît constamment, comme un élément de corruption qui ronge et amenuise la portée de notations dont on connaît, par ailleurs, la vivacité et la richesse ». Lévi-Strauss ([1949b] 1958 et $1974: 26)$.

${ }^{54}$ Voir Lévi-Strauss ([1949b] 1958 et 1974 : 32-39) concernant ses idées sur l'inconscient ethnologique et linguistique.
} 
La fin des cours me permet enfin de souffler. J'ai à vous remercier de votre instructive observation sur mon article de la RHR ${ }^{55}$. Sans doute aurais-je dû dissocier explicitement la légende rapportée par Eschyle, Pindare etc. de l'épisode mythologique, qui me paraît tout différent. Je ne saurais dire comment ni pourquoi les deux se sont associés, mais je doute qu'on puisse donner de l'ensemble une explication unique et cohérente.

J'aurais également dû insister sur l'optique différente sous laquelle nous envisagerons les Suppliantes, selon que nous nous interrogeons sur les intentions d'Eschyle ou sur la motivation réelle de la légende. Il est bien certain que le poète n'a / pas songé à émouvoir les Athéniens avec un drame du cousinage croisé. C'était des « suppliantes » qu'il s'agissait pour lui, du drame des fugitives et de la protection à leur accorder, même au risque d'une guerre, et cela faisait revivre une légende royale que beaucoup devaient connaître. En développant ce thème tout humain, et pour animer son action, Eschyle instaure un débat où, tout naturellement en Attique, le problème est posé tel qu'il devait se poser, quand une fille se trouvait héritière. Là est l'adaptation de la légende au droit du temps. Cela est instructif pour les rapports d'Eschyle avec son temps et son public, mais non pour l'interprétation du drame réel, tel que je le comprends. On ne peut donc voir ici « double jeu » de la part des Danaïdes : elles jouent consciemment l'épiclérat, elles jouent l'endogamie sans le savoir. Toutes / les références que j'ai réunies à l'appui de l'interprétation proposée nous sont données par Eschyle malgré lui. Il n'a certainement pas posé le débat entre l'endogamie et l'exogamie. Mais les traits qu'il rapporte soulignent que tel est bien le fond du problème.

Quand bien même - pour revenir aux termes de parenté - mon étude n'aurait que la valeur d'une position linguistique du problème, je ne croirais pas devoir m'en dispenser. Il s'agit essentiellement de montrer qu'il y a parenté classificatoire jusque peu avant les systèmes historiques. Le reste vous appartient.

Croyez, cher Monsieur, à mes sentiments cordiaux,

EBenveniste

(8)

1 rue Monticelli $14^{\mathrm{e}}$

Le $7 / 2 / 52$

Cher Monsieur,

J'ai lu avec un vif intérêt vos ingénieuses considérations sur l'agencement des éléments dans le vocabulaire des parties du corps en kepkiriwat. ${ }^{56}$ Mais je me sens très embarrassé pour formuler un avis. Non seulement je ne connais de cette langue que ce que vous en citez, mais dans ma lecture je me suis heurté à des difficultés de principe auxquelles je ne sais si une réponse est possible. Je me borne à les énoncer.

La principale est celle-ci : comment analyser les mots ? C'est-à-dire comment analyser les morphèmes ? Il faudrait procéder à une comparaison systématique de ces termes corporels avec le reste du vocabulaire, sans notion préconçue, et voir en particulier selon quels principes s'agencent les éléments identifiables. Il se peut par exemple que des morphèmes qui ont l'air identiques ne puissent l'être en toute position et remplissent selon leur répartition des fonctions

\footnotetext{
${ }^{55}$ Benveniste ([1949] 2015 : 209-216), « La Légende des Danaïdes ». L’article contient ([p. 137n.] p. 215 n.]) une référence aux Structures élémentaires de la parenté.

${ }^{56}$ Les investigations de Lévi-Strauss sur la population kepkiriwát (or kep-kiri-uat, kepikiriwat, Kepi-keri-uate, Quepiquiriuate etc.) de la région rondônienne (du nom du général Rondon) de l'Amazonie brésilienne datent de 1938-1939. Leur langue tupienne occidentale est aujourd'hui éteinte, selon www.ethnologue.com. Lévi-Strauss fait référence à cette population dans plusieurs textes ; voir Lévi-Strauss (1948 et 1950a). Pour le détail de la mission Lévi-Strauss-Vellard voir Loyer (2015 : 199) sv., et Lévi-Strauss (1938 : 384-386).
} 
distinctes : je pense p. ex. à kap qui apparaît <entre> uzabilisep «cils » et uzabilikapsep « sourcil » et qui ici ne se ramène pas facilement à la définition générale indiquée p.13.

Une autre difficulté, connexe d'ailleurs à la précédente, résulte de l'incertitude où l'on est quant à la structure générale de la langue. Y a-t-il des préfixes, des suffixes ? Voici un fait précis. Vous notez une différence singulière entre un groupe de termes, obtenus en indiquant les parties du corps de l'informateur, et un autre groupe qui se rapporte au corps d'animaux dépecés. Ceci me suggère immédiatement une conjecture, que vous avez peut-être envisagée. Cette différence est une différence entre deux préfixes possessifs. Les mots de la première série, commençant tous par $u$-, se réfèrent au corps de l'informateur ; ici « bras » doit signifier « mon bras ». Ceux de la deuxième série, appliqués à un animal, auraient un $s$ - qui serait le possessif de $3^{\mathrm{e}} \mathrm{sg}$. Et ici le mot traduit « estomac » serait «son estomac ». Ce n'est qu'une hypothèse, mais a priori les noms de parties du corps, obtenus de cette manière, doivent être munis d'indices de possession. Ceci modifierait passablement l'interprétation des termes. /

Il y a aussi dans ce vocabulaire des discordances : pour «cils » on trouve à la fois unzapisep (p. 11, 12) et undjakapsep (p.13), sans différence ? - Dans les formes énumérées p.7, je soupçonne que les traductions doivent être rectifiées : sur quoi repose l'interprétation d'une série de formes par des infinitifs, d'une autre par un impératif (tisser / pose-le ...), quand la finale est identique ? A-t-on des raisons d'écrire en un mot umambikutangen et en deux unanbu kutanga? Et d'une manière générale ces formes ont-elles été obtenues dans des conditions qui permettent d'exclure des expressions complexes quand on attendait des termes simples ? par exemple, l'informateur a-t-il donné «poing» simplement ou «je ferme le poing »?

Il est fort possible qu'il y ait à la base d'un grand nombre de ces termes, comme vous le supposez, certains procédés de composition analogues à ceux du guato. C'est à des problèmes préliminaires que je m'arrête, car toute l'analyse en dépend, et je ne le fais que pour assurer les bases d'une interprétation. Il faudrait en tout cas un examen de tous les faits recueillis pour déterminer les classes de formes (nominales, verbales, simples, composées, suffixées, etc.) et procéder plus sûrement à l'analyse de ces termes.

Croyez toujours, cher Monsieur, à mes sentiments cordialement dévoués, EBenveniste

Cher Monsieur,

\section{Le 11 Février 1952}

Surtout ne croyez pas que mes observations impliquent une critique du procédé de représentation que vous proposiez ${ }^{57}$. Simplement une réserve sur la possibilité de justifier une analyse morphologique rigoureuse. Je me suis donc tenu très en deçà du plan où vous portiez la discussion, en me bornant à voir si le matériel lexical (dont je sais dans quelles conditions

\footnotetext{
${ }^{57}$ Lévi-Strauss semble ne pas avoir apprécié les critiques de Benveniste énoncées dans la lettre précédente, à tel point qu'il suspend la publication d'un article. Il confie à Roman Jakobson dans une lettre du 13 mars 1952 : « Je suis d'autant plus agacé par cette polémique [polémique de chercheurs concernant son article « Language and the Analysis of Social Laws » (1951)] qu'ayant récemment terminé mon étude sur les noms des parties du corps dans les langues sud-américaines dont vous aviez aussi approuvé l'esquisse je l'ai montrée à Benveniste, qui l'a complétement condamnée. Or, aussi défectueux que soient le matériel et naïve ma méthode, il y avait tout de même dedans quelque chose de curieux : c'est que, dans les langues considérées, les termes paraissent formés par une combinaison de morphèmes, laquelle paraît interprétable en chaînes de Markov. Même si ce n'est pas vrai, il me semble que l'hypothèse méritait d'être vérifiée ; et j'avais signalé à Benveniste qu'une personne compétente pouvait le faire sur l'algonkin, où les mots sont manifestement construits par le même procédé. Devant l'attitude critique de Benveniste, j'ai renoncé à envoyer le manuscrit à l'impression ». Voir. Roman Jakobson et Claude Lévi-Strauss (2018 : 149-150).
} 
difficiles il a été recueilli) permettait d'identifier à coup sûr les éléments en combinaison. Il serait néanmoins intéressant de vérifier votre interprétation dans une langue beaucoup mieux connue, comme l'algonkin, où l'analyse est assurée pour l'essentiel. Vous en auriez l'occasion en travaillant cette année auprès de Voegelin ${ }^{58}$.

J'ai décliné une proposition américaine pour l'été prochain. Si j'y allais, je voudrais pouvoir enquêter chez les Indiens, mais c'est inconciliable avec une période d'enseignement, et j'ai beaucoup à faire ici.

Croyez-moi, cher Monsieur, votre cordialement dévoué, EBenveniste

$14 / 11 / 53$

Au cours de mon enquête ${ }^{59}$, qui a été longue et dure et assez fructueuse, je n'ai pas perdu de vue la question qui vous intéressait. Chez les Tlingit, pas de réaction caractérisée au corbeau zoologique. Mais en athapaske du nord, chez les «Loucheux» du Haut Yukon, un indice curieux : le corbeau est nommé descriptivement " excréments autour de la bouche ${ }^{60}$ ", car, disent ces chasseurs-trappeurs, le corbeau ne vit que de charogne, il est incapable de tuer quelque être que ce soit. Cela n'empêche pas le corbeau mythologique d'avoir l'importance que vous savez.

Croyez, cher Monsieur, à mes sentiments cordiaux,

EBenveniste

1 rue Monticelli $14^{\mathrm{e}} \quad$ Le 20 Déc. 55

Cher Monsieur,

J'ai commencé votre livre ${ }^{61}$ pendant les heures lucides que me laissait une récente convalescence, et d'une page à l'autre, d'un chapitre au suivant, j'ai retenu longtemps, pour arriver au bout d'une lecture captivante, le remerciement que je vous devais. Et maintenant je ne saurais plus louer comme il faudrait ce livre si riche, où tous les genres se mêlent, où les pages les plus désintéressées vibrent d'un accent personnel. On voudrait commenter à chaque page tout ce que vous offrez à foison, les descriptions ou les méditations. Mais on se sent entraîné à imaginer, au delà de ce qu'on lit, le destin possible d'un ouvrage qui ouvre tant de problèmes. La belle et haute méditation où il s'achève éclaire un aspect de notre civilisation qui n'apparaît si lucidement que parce qu'il en présage la fin probable, et peut-être votre livre hâtera-t-il une prise de conscience qui précipitera cette fin. Vous avez enrichi la littérature ethnographique d'un témoignage qui retentira dans la conscience des ethnographes et sans doute la transformera. On regrette seulement qu'un livre pareil risque d'être confondu - en partie à cause d'un titre qui ne me semble pas des plus heureux - avec tant de relations qu'il dépasse de si loin. Mais j'ai confiance que ceux qui sont aptes à le lire sauront le découvrir, et non pas seulement en France. /

\footnotetext{
${ }^{58}$ Charles Voegelin (1906-1986), linguiste et anthropologue américain, professeur à l'université d'Indiana à partir de 1947, éditeur de l'International Journal of American Linguistics à partir de 1944, après la mort de F. Boas. Auteur de nombreux articles et monographies sur les langues amérindiennes de l'Amérique du Nord. ${ }^{59}$ Il s'agit du second séjour d'enquête d'Émile Benveniste sur la côte Nord-Ouest américaine, durant lequel il travaille principalement sur let Tlingit, mais aussi le Gwich'in (Loucheux) et l'Inupiak.

${ }^{60}$ Voir Benveniste ([1953] 2015). Pour l'analyse du nom du corbeau, voir p. 237.

${ }^{61}$ Lévi-Strauss (1955), Tristes tropiques.
} 
- J'ai bien reçu votre lettre au sujet de l'Homme. L'arrangement que vous envisagez avec Plon me parait heureux, puisqu'il sauvegarde la nature scientifique de la collection, tout en lui assurant une diffusion meilleure, et je suis heureux que vous ayez obtenu un crédit appréciable pour faire repartir la Collection. Je souhaite que les manuscrits retenus soient de qualité - mais votre choix en est garant. Je vous donne donc mon accord pour que l'Homme passe chez Plon aux conditions que vous mentionnez.

Croyez-moi, cher Monsieur, votre amicalement dévoué,

EBenveniste

PS. Je suis affligé d'un œil qui ne peut rien sauter dans une lecture. J'espère que, pour une nouvelle édition de votre livre, une gracieuse Nambikwara consentira à dévorer la dernière lettre du «poux » (p. 298 fin), et que vous transfériez à « sans que je m'aperçus » (p. 315) l'appendice gênant de "se départissait» (p. 284). Est-ce pour préparer la tâche d'un futur traducteur qu'il y a un anglicisme p. 357 «il s'exprime par et trouve ... dans un jeu ...»? Excusez ces vétilles.

1 rue Monticelli $14^{\mathrm{e}}$

Le 10/10/56

Cher Monsieur,

J'ai à vous remercier de l'envoi de votre remarquable étude sur les organisations dualistes ${ }^{62}$, qui pose un important problème en termes qui le rendent non seulement accessible, mais instant pour d'autres que les sociologues aussi. J'espère que cette vigoureuse démonstration suscitera en anthropologie un débat - où je me garderai bien d'entrer. Mais depuis que je vous ai lu, je suis en proie à des perplexités que, faute de connaître d'assez près les faits que vous articulez, je ne saurais même formuler exactement. Voici le type de questions que je me pose : $1^{\circ}$ ) est-il vraiment possible de réduire l'un à l'autre les systèmes qui commandent les organisations diamétrales et concentriques respectivement ? La notion de centre, et de cercle autour d'un centre, me semble quelque chose d'hétérogène et d'irréductible ; il détermine un autre type de structure logique, qui ne répond pas et ne se ramène pas à la structure bipartie des organisations dualistes - ni inversement. $-2^{\circ}$ ) La division établie sur l'opposition : proximité / éloignement du centre ne paraît pas avoir le même sens que la division : possibilité / impossibilité du mariage ; $3^{\circ}$ ) Les organisations triadiques, d'après vos exemples, peuvent fort bien coexister dans une société avec une structure dualiste ; elles n'ont pas le même objet ; est-il donc nécessaire d'imaginer que ce dualisme est toujours un triadisme dégénéré ou simplifié ? 4º Pourquoi (p. 119-120) les oppositions du type «logiquement hétérogènes" ("stabilité / changement», etc.) seraient-elles moins recevables dans la symbolique sociale que celles de gauche /droite, été / hiver - . Au fond, guerre et paix ; création et conservation du monde ; cru et cuit sont <termes $>$ aussi complémentaires que haut et bas, aîné et cadet. 5) Ceci en marge de votre argumentation et sans rapport précis avec votre raisonnement : je me demande s'il ne serait pas possible de découvrir dans une relation triadique concrète, identifiable sur un point quelconque du monde, / entre deux termes opposés et complémentaires, un troisième qui serait la «neutralisation» de l'opposition. Si c'est concevable et démontrable, ce que je ne sais, ce serait une trouvaille de grande portée théorique, et qui en outre, concilierait d'un coup dualisme et triadisme.

Peut-être n'ai-je pas su voir que toutes ces questions avaient déjà leur réponse dans vos pages ? Ne doutez pas en tout cas de l'intérêt que j'ai pris à vous lire.

${ }^{62}$ Lévi-Strauss ([1956] 1958), « Les organisations dualistes existent-elles ? ». 
- J'en viens maintenant à votre note au sujet de L'Homme. Mon accord vous est acquis, puisque vous avez retenu ces mss. Une seule observation : est-il prudent de publier des plaquettes dans cette série ? Car plaquettes deviendront des mss. de 30 p. dactyl., donc à peine 18-20 pages d'impression large, comme les 3 derniers mentionnés. Ce serait admissible pour des écrits doctrinaux (j'aurais très bien vu votre article publié ou republié* $<$ [en marge] *oui, pourquoi pas ?> sous cette forme), moins pour des contributions spécialisées. Ne vaudrait-il pas mieux en grouper deux ou trois - qui auraient quelque affinité - sous une même couverture ? Je pose simplement la question, qui est d'ordre pratique.

Croyez-moi, cher Monsieur, votre bien cordialement dévoué,

EBenveniste

(13)

Collège de France

Chaire de Grammaire comparée

Paris, le $3 / 2 / 59$

1 rue Monticelli $14^{\mathrm{e}}$

Cher Monsieur,

J'apprends avec satisfaction que la chaire est créée ${ }^{63}$; cette fois la création s'est faite, me semble-t-il, dans des délais assez courts, mais naturellement il ne sera pas question d'une nouvelle visite ${ }^{64}$. Aucun problème ne devrait surgir maintenant, et la seconde étape vous acheminera au but.

- Il serait dommage de renoncer à éditer l'Homme en France ; je ne sais rien de l'aspect commercial du problème, mais il faudrait essayer de tenir, au moins jusqu'à ce que / le CNRS, qui répugne, on le sait, à s'engager pour longtemps, vous ait fait connaître sa décision. cordiaux,

Vous n'avez plus besoin de souhaits. Je vous dis seulement mes sentiments bien

\section{EBenveniste}

PS. Je reçois à l'instant les papiers de Sol Tax au sujet de Current Anthrop. ${ }^{65} \mathrm{~J}$ 'hésite un peu à m'engager dans cette nouvelle entreprise qui semble conçue pour devenir accaparante.

Collège de France

Paris, le 26 Avril 59

Chaire de Grammaire comparée

Cher Monsieur,

C'est avec retard que je vous réponds. J'ai passé trois semaines à l'hôpital à me délivrer d'une sorte de grippe infectieuse, et quelques jours de convalescence dans le Midi. Veuillez m'excuser de me borner aujourd'hui à la question la plus urgente : celle de l'Homme. (Votre communication antérieure était restée en souffrance ici).

Je déplore le tour qu'a pris la correspondance entre Plon et le CNRS, et j'approuve tout à fait la ligne de conduite / que vous avez adoptée. Il faut que nous restions maîtres de publier ce que nous jugeons notable, et donc que le soutien accordé à la collection reste global. Je

\footnotetext{
${ }^{63}$ Chaire d'Anthropologie sociale, créée par l'assemblée générale des professeurs au Collège de France le 30 novembre 1958. Lévi-Strauss sera élu lors de l'assemblée du 15 mars 1959 (voir Loyer 2015 : 447-448).

${ }^{64}$ Selon la tradition, les candidats à une chaire au Collège de France doivent effectuer des visites auprès de chaque professeur.

${ }^{65}$ L'anthropologue américain Sol Tax (1907-1995) a fondé la revue Current Anthropology
} 
souhaite que vous puissiez aboutir dans ces nouvelles démarches ; il nous faudrait un éditeur un peu hardi, mais pas tellement, car finalement cela paraît se vendre.

Je veux vous remercier de vos observations sur mes articles, mais y mieux réfléchir avant de vous répondre. Vos remarques sont de grande importance. Croyez-moi votre bien cordialement dévoué,

\section{EBenveniste}

15)

Collège de France

Chaire de Grammaire comparée
Paris, le 16 Février 62

1 rue Monticelli $14^{\mathrm{e}}$

Cher ami,

Merci pour votre message. Chez moi il n'y a que des dégâts matériels, portes arrachées, entrée ravagée. A un mètre près, j'ai échappé dans mon bureau, à la projection des portes. Mais je n'ai pas éprouvé de commotion sérieuse ${ }^{66}$.

Tout cela sera, je pense, bientôt réparé. Votre sympathie m'est précieuse.

Votre aimablement dévoué,

EBenveniste

(16)

Collège de France

Paris, le 18 juin 62

1 rue Monticelli $14^{\mathrm{e}}$

Cher ami,

Permettez-moi, ayant lu vos deux livres à la suite ${ }^{67}$, de les englober dans un même remerciement. J'ai suivi avec admiration ces deux exposés complémentaires, le premier critique et réfutant le totémisme par ses contradictions, le second synthétique et intégrant le totémisme dans une vaste classification. Cette analyse des classifications ou de la pensée classificatoire en ses multiples aspects abonde en observations importantes et alimentera certainement des recherches nouvelles. J'ai été personnellement très séduit par vos observations / sur la nature classificatoire des noms propres ${ }^{68}$, comme à un autre point de vue, j'ai trouvé une vive saveur à vos remarques sur la pensée de Bergson ${ }^{69}$.

Je vous félicite de fournir ainsi de belles réinterprétations des problèmes classiques de l'anthropologie et une contribution de valeur à l'analyse de la culture.

Croyez-moi bien votre amicalement dévoué,

EBenveniste

\footnotetext{
${ }^{66}$ Cette lettre fait référence aux attentats de l'OAS à Paris et dans la région parisienne du mercredi 7 février 1962 : dix plasticages à la porte du domicile parisien d'hommes politiques, d'intellectuels, de professeurs, de journalistes, voir entre autres Le Monde, daté 9 février 1962. Il n'est pas certain que Benveniste ait été personnellement visé. Il se peut que son appartement ait été endommagé par un plasticage qui eut lieu sur le même palier. L'immeuble du 1, rue Monticelli était (et est encore) la résidence de nombreux universitaires.

${ }^{67}$ Claude Lévi-Strauss ([1962a] 2002), Le totémisme aujourd'hui et (1962b), La pensée sauvage.

${ }^{68}$ Cf. Lévi-Strauss (1962b : 202-259).

${ }^{69}$ Voir Lévi-Strauss [1962a] 2002 : 136 sv., concernant la discussion des thèses d'Henri Bergson sur le totémisme telles qu'énoncées dans Les Deux Sources de la morale et de la religion (1932) et son rapprochement avec les conceptions de Radcliffe-Brown.
} 
(17)

Collège de France

Chaire de Grammaire comparée
Paris, le 21 Nov. 62

1 rue Monticelli $14^{\mathrm{e}}$

Cher ami,

Je suis content que mon livre ne vous ait pas semblé trop rebutant ${ }^{70}$. Il est si difficile en ce domaine de dépasser le niveau de la pure technique où l'on se confinait, et d'atteindre celui des vrais problèmes.

Vous en avez discerné un, et fort justement : en effet antiyant- " gendre » témoigne pour la matrilocation. Mais dans la complexité de la culture hittite où des influences divines se croisent, l'absence de la formule «conduire (la femme ${ }^{71}$ )» pourrait n'être pas /encore à expliquer par là. Cela semble néanmoins fort probable, et un hittitologue m'a confirmé, depuis, que la seule expression usitée est «prendre en mariage».

Merci, et croyez à mes sentiments amicaux,

EBenveniste

(18)

Collège de France

Chaire de Grammaire comparée

\author{
Paris, le 9 février 64 \\ 1 rue Monticelli $14^{\mathrm{e}}$
}

Cher ami,

Tous mes remerciements pour le précieux dépouillement ethnologique que vous avez si aimablement fait faire à mon intention. Les résultats, en effet, ne sont pas absolument concordants ; néanmoins la proportion des coïncidences entre matrilocation et teknonymie est plus élevée que je ne l'aurais cru, et pourrait suggérer une relation entre les deux phénomènes. Mais je me garderais de rien affirmer. /

J'ai eu une conversation avec Cuisenier ${ }^{72}$, et il m'a fait part des améliorations qu'il avait apportées à son article ${ }^{73}$ après l'avoir fait relire par des turcologues. Si vous jugez satisfaisante cette nouvelle rédaction - que je n'ai pas vue -, on pourrait la prendre pour l'Homme.

Laissez-moi vous féliciter pour votre récente promotion ${ }^{74}$, que je viens d'apprendre, et y joindre tous les bons vœux de

Votre amicalement dévoué,

EBenveniste

(19)

Collège de France

Chaire de Grammaire comparée
Paris, le 26 Nov. 64

1 rue Monticelli $14^{\mathrm{e}}$

Cher ami,

\footnotetext{
${ }^{70}$ Benveniste (1962), Hittite et indo-européen : Études comparatives. Sur hitt. antiyant-, voir spécialement p. 12, glosé par Benveniste comme 'qui entre dans la famille du beau-père'.

${ }^{71}$ Allusion à la formule indo-européenne reflété par lat. uxorem ducere, cf. Benveniste (1969a : 240-241), dans le chapitre «L'expression indo-européenne du mariage ».

72 Jean Cuisenier (1927-2017) est un ethnologue français, spécialiste des traditions populaires, en particulier de l'architecture rurale. En 1971, il soutient une thèse, Économie et parenté, leurs affinités de structure dans le domaine turc et dans le domaine arabe sous la direction de Raymond Aron.

${ }^{73}$ Cuisenier (1962).

${ }^{74}$ Il pourrait s'agir de la Légion d'honneur, au grade d'officier. Claude Lévi-Strauss est fait Grand Officier de la Légion d'honneur en 1985, et Grand Croix en 1991.
} 
J'ai un peu tardé à vous remercier de votre envoi. C'est que j'ai mis du temps à lire Le Cru et le Cuit. ${ }^{75} \mathrm{Il}$ faut lire ce livre ou très vite et d'un trait, ce que je ne pouvais faire, ou à petites étapes et en rêvant un peu, ce que j'ai fait, et sans me flatter d'avoir pu suivre de tout point vos exégèses, je suis émerveillé de l'ingéniosité et de la rigueur que vous apportez à découvrir - puis à interpréter dans des connexions systématiques, les éléments signifiants des mythes. Je pense en particulier à tout le développement sur cuisine et bruit, mais il faudrait s'arrêter à maint autre endroit. C'est un ouvrage d'une richesse très singulière, où s'entrelacent tant d'appels et d'échos que, indépendamment des références musicales qui l'encadrent, je pensais sans cesse à une composition de Messiaen, à tort ou à raison, je ne sais. /

En tout cas vous avez promu l'étude du mythe à un plan tout nouveau et qui annonce une transformation de votre méthode d'analyse.

Tous mes remerciements pour ce beau livre et croyez à mes sentiments amicaux,

EBenveniste

(20)

Collège de France

Chaire de Grammaire comparée
Paris, le 14 Février 1967

1 rue Monticelli $14^{\mathrm{e}}$

Cher ami,

Je termine la lecture captivante de votre livre ${ }^{76}$ où, à l'ombre de Virgile et de Rousseau, les fumées de tabac et les rayons de miel engendrent une étonnante richesse de mythes. Plus captivante encore est votre analyse des structures apparentes ou implicites où cette richesse s'organise et devient signifiante. Vous avez accompli une nouvelle étape sur la voie que vous vous êtes assignée. Le linguiste vous suit dans vos démarches, voit les analogies et les différences avec sa propre pratique, et les perspectives, diverses aussi, qui s'ouvrent. Dans certains de vos développements la langue intervient directement que ce soit dans la forme du discours ou dans les termes du vocabulaire, et la réflexion en est vivement stimulée.

J'aurais souhaité, mais peut-être n'était-ce pas possible, que le domaine de l'enquête fût circonscrit par des critères extérieurs à la matière de l'enquête, par exemple par la relation des langues entre elles, ou une certaine symbiose culturelle, etc., et qu'on pût ainsi distinguer entre identités ou affinités d'une part, / homologies de l'autre. Cela aurait été de grande portée méthodologique. Vous avez indiqué çà et là des analogies avec des domaines non contigus au vôtre : Pacifique N.O. ou Chine. Des distinctions pourraient-elles être tracées à l'intérieur du monde indien tropical ? Il s'agirait de mieux voir s'il y a des enchaînements nécessaires et à quelles conditions.

Je souhaite qu'un jour vous puissiez étudier parallèlement tout ce grand complexe mythique du miel et de l'ours dans les traditions nordiques et sibériennes - ou encore les relations structurales de la boiterie dans d'autres mythologies (Hephaistos !). Mais on n'en finirait pas, tant cette lecture suscite de questions et éclaire d'analogies. C'est à tous égards un livre important.

- Beaucoup moins l'est probablement celui ${ }^{77}$ que je vous envoie, en souvenir de cette si agréable soirée où, entre autres, j'ai appris que les proverbes éveillaient la curiosité de votre jeune fils ${ }^{78}$.

\footnotetext{
${ }^{75}$ Lévi-Strauss (1964), Le cru et le cuit.

${ }^{76}$ Lévi-Strauss (1967), Du miel aux cendres.

${ }^{77}$ Benveniste a publié deux livres en 1966 : Problèmes de linguistique générale et Titres et noms propres en iranien ancien. Il semblerait assez prévisible qu'il ait considéré le premier comme spécialement destiné à LéviStrauss, dans le contexte du «structuralisme » parisien de l'année 1966.

${ }^{78}$ Matthieu, né en 1957 de l'union de Claude Lévi-Strauss avec Monique Roman.
} 
Merci pour ce nouvel enrichissement et croyez à mes amitiés, EBenveniste

(21) [Remarques adressées par Benveniste à Lévi-Strauss en octobre $1961^{79}$ concernant l'article sur « Les Chats » de Baudelaire $\left.{ }^{80}\right]$.

\section{QUELQUES REMARQUES}

1) D'après l'éd. de Sacy, il y a une variante. Aux vv. 7-8, le texte du Corsaire porte : l'Erèbe les eût pris ... s'il pouvait ... Cela mériterait mention, par ex. p.23.

2) Dans l'analyse des rimes, il faudrait considérer aussi ce « système interne » des demivers :

$1 \tilde{\mathrm{a}}----\varepsilon r$

2 ã $----\tilde{o}$

$3 \mathrm{u}----\tilde{\mathrm{o}}$

$4 \varnothing---\varepsilon r$

3) p. 19 le dernier alinéa n'est pas clair. Est-ce intentionnellement qu'on parle ici (et ici seulement) de « lignes » au lieu de « vers »?

4) Il y a quelques flottements dans ces pages, me semble-t-il, sur le statut de l' « Erèbe ». Il est partout pris comme inanimé (cf. p.20, 23), mais aussi parfois comme un être (p.25, 32). Une personnification ne peut être réduite à l'inanimé : d'ailleurs l'Erèbe est frère de la Nuit. Ou alors il faudrait instituer un "genre » spécial pour les êtres mythiques, ce qui serait admissible ici.

5) Un motif n'est pas relevé dans cette analyse, c'est celui de la « mûre saison ». Il m'a toujours paru significatif : de Baudelaire dans sa notion de la beauté, et de ce sonnet. La « mûre saison » est médiatrice entre « amoureux fervents » et « savants austères » : c'est dans leur mûre saison qu'ils se rejoignent, pour s'identifier « également» aux chats. Car rester « amoureux fervents » jusque dans la « mûre saison » signifie déjà qu'on est hors de la vie commune, tout comme sont les « savants austères » par vocation. La situation initiale du sonnet est celle de la vie hors du monde (néanmoins la vie souterraine est refusée) et elle se développe, transférée aux chats, de la réclusion frileuse vers les grandes solitudes étoilées où science et volupté sont rêve sans fin. /

Baudelaire, Euvres complètes, éd. S. de Sacy, Paris, Club du Meilleur Livre, 1955, tome I, p. 1227 [notes sur Les Chats] :

14 novembre 1847, Le Corsaire (dans le feuilleton de Champfleury : « Le Chat Trott $\gg)$.

1848, Revue de Belgique (p.280-281, dans un article de Retchezken ${ }^{81}$ ).

Str. II, v.3-4. Ils présentent un sens différent selon que l'on donne au verbe prendre son sens propre ou le sens figuré de considérer, regarder comme, ce dernier sens s'accordant mieux

\footnotetext{
${ }^{79}$ Manuscrits conservés dans le fonds Lévi-Strauss, dans le dossier NAF 28150 (67). Voir Jakobson et LéviStrauss (2018: 243-245) et Testenoire 2019, lettre 12.

${ }^{80}$ Jakobson et Lévi-Strauss (1962: 5-21).

81 «Retchezken est un pseudonyme utilisé par trois auteurs belges lorsqu'ils écrivent conjointement : Léon Jouret (1828-1905), musicologue et compositeur, Léon Gauchez (1825-1907), critique et marchand d'art, et Edouard Wacken (1819-1861), dramaturge, poète et critique, et directeur de la Revue de Belgique, dans laquelle ces textes paraissent ». (Jakobson et Lévi-Strauss 2018 : 244 n.2)
} 
avec la syntaxe de cette phrase. Remarquons d'ailleurs que Le Corsaire et la Revue de Belgique font écrire à Baudelaire :

$S$ 'il pouvait au servage incliner leur fierté.

désignant ainsi l'Erèbe.

Voilà la source de mon observation, d'après une édition que vous pouviez ne pas avoir sous la main. La variante aurait donc été donnée deux fois.

Veuillez croire à mes sentiments amicaux.

E. Benveniste

\section{Bibliographie}

Archives de la Rockefeller Foundation. RF, RG 1.2. Series 500R. Box 12. f. 112.

Fonds Claude Lévi-Strauss. Départements des Manuscrits. Bibliothèque nationale de France. NAF 28150.

Bader, Françoise. 2012. « Lettres d'Émile Benveniste à Claude Lévi-Strauss. Contribution à la biographie d'Émile Benveniste ». In : Giampaolo Borghello e Vincenzo Orioles (a cura di), Per Roberto Gusmani 1. Linguaggi, culture, letterature 2. Linguistica storica e teorica. Studi in ricordo. Udine : Forum. 227-249. Disponible en ligne. URL : http://forumeditrice.it/percorsi/lingua-e-letteratura/studi-in-onore/per-robertogusmani/lettres-d2019emile- benveniste-a-claude-levi consulté le 18/04/2020.

Bartholomae, Christian. 1904. Altiranisches Wörterbuch. Strassburg : Karl J. Trübner.

Beekes, S.P. Beekes. 2010. Etymological dictionary of Greek. With the assistance of Lucien van Beek. Leiden-Boston: Brill.

Benveniste, Émile. [1932] 2015. «Les classes sociales dans la tradition avestique ». Langues, Cultures, Religions. 47-59.

— [1938] 2015. «Traditions indo-iraniennes sur les classes sociales ». Langue, cultures, religions. 105- 118.

— [1949] 2015. « La légende des Danaïdes ». Langues, Cultures, Religions, Limoges : Lambert-Lucas. 209-216.

— [1953] 2015. « Le vocabulaire de la vie animale chez les Indiens du Haut Yukon (Alaska) ». Langues, cultures, religions. Limoges : Lambert-Lucas. 225-250.

- 1962. Hittite et indo-européen : Études comparatives. Paris : Adrien-Maisonneuve.

— 1965. « Termes de parenté dans les langues indo-européennes. L’Homme 5(3-4) : 5-16. Juillet-décembre 1965. Études sur la parenté.

https://journals.openedition.org/lhomme/persee-123934 consulté le 18/04/2020.

- 1966. Problèmes de linguistique générale. Paris : Gallimard.

- 1974. Problèmes de linguistique générale 2. Paris : Gallimard.

- 1969a. Le vocabulaire des institutions indo-européennes. 1. Économie, parenté, société. Paris : Minuit.

- 1969b. Le vocabulaire des institutions indo-européennes. 2. Pouvoir, droit, religion. Paris : Minuit.

— 2011. Baudelaire. Edition, introduction et notes par Chloé Laplantine. Limoges : Lambert-Lucas.

- 2015. Langues, cultures, religions. Choix d'articles réunis par Chloé Laplantine et Georges-Jean Pinault. Limoges : Lambert-Lucas.

Boas, Franz. 2018. Introduction du Handbook of American Indian languages (1911). Traduction d'Andrew Eastman et Chloé Laplantine. Préface de Chloé Laplantine. Limoges : Lambert-Lucas. 
Bruneau, Michel. 2000. «Pierre Gourou (1900-1999)». L'Homme [En ligne] 153 | janviermars 2000, URL : http://journals.openedition.org/lhomme/1 consulté le 18/04/2020.

Capell, Arthur. 1949. « The Concept of Ownership in the Languages of Australia and the Pacific », Southwestern Journal of Anthropology, 5(3) : 169-189.

Chantraine, Pierre. 1968-1980. Dictionnaire étymologique de la langue grecque. Histoire des mots. Paris : Klincksieck.

Cuisenier, Jean. 1962. «Endogamie et exogamie dans le mariage arabe ». L'Homme 2(2) : 80-105.

Garvin, Paul A. Ponapean, a Micronesian Language. [texte dactylographié]

Hauser, Stefan R. 2003 (updated March 2012). « Ernst Herzfeld. i. Life and work ». In: Ehsan Yarshater (ed.), Encyclopcedia Iranica. XII/3, New York, Bibliotheca Persica Press, 2003. Disponible en ligne, URL :

http://www.iranicaonline.org/articles/herzfeld-ernst-i-1 consulté le 18/04/2020.

Herzfeld, Ernst. 1947. Zoroaster and His World, t. 1. Princeton : Princeton University Press.

Jakobson, Roman et Lévi-Strauss, Claude.1962. " "Les Chats” de Charles Baudelaire.

L'Homme 2(1) : 5-21.

- 2018. Correspondance 1942-1982. Préfacé, édité et annoté par Emmanuelle Loyer et Patrice Maniglier. Paris : Seuil. Librairie du XXI ${ }^{\mathrm{e}}$ siècle.

Laplantine, Chloé. 2011. Emile Benveniste, l'inconscient et le poème. Limoges : LambertLucas.

— 2018. « Préface ». In: Franz Boas. Introduction du Handbook of American Indian languages (1911). Limoges: Lambert-Lucas.

Lévi-Strauss, Claude. [1945] 1958 et 1974. «L'analyse structurale en en linguistique et en Anthropologie ». Anthropologie structurale. Paris : Plon : 43-69.

— 1948. « Tribes of the Right Bank of the Guaporé River ». In: Julian H. Steward (ed.). Handbook of South American Indians, vol. 3: The tropical forest tribes. Bureau of

American Ethnology. Bulletin 143. Washington, D. C. : Smithsonian Institution. 371379.

— [1949a] 1967. Les structures élémentaires de la parenté. Berlin-New York, Mouton de Gruyer.

— [1949b] 1958 et 1974. « Histoire et ethnologie ». Anthropologie structurale. Paris : Plon.

- 1950a. «Documents Rama-rama ». Journal de la Société des Américanistes 39 : 73-84.

— 1950b. «Introduction à l'œuvre de Marcel Mauss ». In : Marcel Mauss. Sociologie et anthropologie. Paris : PUF. IX-LII.

- 1951. « Language and the Analysis of Social Laws ». American Anthropologist 53(2).

155-163. (Adaptation française : «Langage et société ». Anthropologie structurale).

- 1955. Triste tropiques. Paris : Plon. Mythes et religions.

— [1956] 1958. « Les organisations dualistes existent-elles? ». Anthropologie structurale. Paris : Plon. 147-182.

— [1962a] 2002. Le totémisme aujourd'hui. Paris : Presses Universitaires de France.

[Disponible en ligne. URL : https://www.cairn.info/le-totemisme-aujourd-hui-9782130528784.htm?contenu=presentation consulté le 18/04/2020).

- 1962b. La pensée sauvage. Paris : Plon.

- 1964. Le cru et le cuit (Mythologiques, 1). Paris : Plon.

- 1967. Du miel aux cendres (Mythologiques, 2). Paris : Plon.

— 1976. « Hommage à Émile Benveniste ». L'Homme 16(4) : 5.

- 1991. Histoire de Lynx. Paris : Plon.

Lévi-Strauss, Dina. 1938. « Mission Lévi-Strauss-Vellard (1938-1939) ». Journal de la Société des Américanistes 30(2) : 384-386.

Loyer, Emmanuelle. 2015. Claude Lévi-Strauss. Paris : Flammarion. 
Mayrhofer, Manfred. 1956-1976. Kurzgefasstes etymologisches Wörterbuch des Altindischen. 3 tomes. Heidelberg : Carl Winter.

- 1986-1996. Etymologisches Wörterbuch des Altindoarischen. 1. Teil : Ältere Sprache. 2 tomes. Heidelberg : Carl Winter.

Meillet, Antoine. [1906] 1921. "L'état actuel des étudies de linguistique générale ». Linguistique historique et linguistique générale [I]. Paris : Champion. 1-18. Réimprimé 1982. Genève : Slatkine. Nouvelle édition préparée par Pierre Ragot : 2015. Linguistique historique et linguistique générale. Limoges : Lambert-Lucas. 7996.

Morgenstierne, Georg. 1927. An etymological vocabulary of Pashto. Oslo: Jacob Dybwad.

- 2003. A new etymological vocabulary of Pashto. Compiled and edited by J. Elfenbein, D.N. MacKenzie and Nicholas Sims-Williams. Wiesbaden: Reichert.

Renou, Louis. 1961. Anthologie sanskrite. Textes de l'Inde ancienne traduits du sanskrit. Paris : Payot

Sauvageot, Antoine. 1951. Compte rendu de : Garvin Paul L. Ponapean, a Micronesian Language. Journal de la Société des Océanistes 7 : 315-317.

Skjaervø, Oktor Skjaervø. 2013. « Marriage ii. Next-of-kin marriage in Zoroastrianism ». Encyclopcedia Iranica, online edition, 2013, URL : http://www.iranicaonline.org/articles/marriage-next-of-kin consulté le 18/04/2020).

Szemerényi, Oswald. 1977. Studies in the kinship terminology of the Indo-European languages. = Acta Iranica 16. Textes et mémoires. Vol. VII: Varia 1977. TéhéranLiège: Bibliothèque Pahlavi \& Leiden : Brill.

Testenoire, Pierre-Yves. 2019. « Compléments à la correspondance Jakobson - Lévi-Strauss ». Acta structuralica 4. URL : https://doi.org/10.19079/actas.2019.4.2 consulté le $18 / 04 / 2020$. 